\title{
Targeting $\mathrm{HIBCH}$ to reprogram valine metabolism for the treatment of colorectal cancer
}

\author{
Yunlong Shan', Yuan Gao', Wei Jin' Minmin Fan', Ying Wang², Yanhong Gu², Chenxiao Shan ${ }^{3}$, Lijun Sun ${ }^{4}$, Xin Li', \\ Biao $\mathrm{Yu}^{4}$, Qiong Luo ${ }^{1}$ and Qiang $\mathrm{Xu} \mathbb{B}^{1}$
}

\begin{abstract}
Valine catabolism is known to be essential for cancer cells but the detailed mechanism remains unclear. This study is to explore the critical roles of 3-hydroxyisobutyryl-CoA hydrolase $(\mathrm{HIBCH})$ in colorectal cancers (CRC) and to develop a new therapy returning valine metabolism homeostasis. High $\mathrm{HIBCH}$ expression was first confirmed to correlate with poor survival in patients with CRC, which was then linked to the increased cell growth, resistant apoptosis, and decreased autophagy in CRC cells. The functions of $\mathrm{HIBCH}$ in CRC were dependent on its mitochondrial localization. High HIBCH level was further demonstrated to promote the metabolism of tricarboxylic acid cycle as well as oxidative phosphorylation in CRC cells. Based on above findings, we further discovered a novel valine catabolism inhibitor SBF-1. The pharmacological blockade of HIBCH mitochondrial localization with SBF-1 resulted in decreased cancer cell growth and increased autophagy, collectively contributing to the antitumor effect both in vitro and in vivo. Moreover, anti-VEGF therapy with bevacizumab increased HIBCH level in CRC cells, which in turn caused the resistance to the therapy. The interference with HIBCH function by SBF-1 significantly increased the antitumor efficacy of bevacizumab and led to a robust survival benefit. The present study identified $\mathrm{HIBCH}$ as a critical enzyme of valine catabolism in CRC progression and resistance to anti-VEGF therapy. We also provided a novel HIBCH inhibitor SBF-1, which highlighted the combined therapy using valine catabolic inhibitor along with anti-VEGF drugs, to control progression of CRC.
\end{abstract}

\section{Introduction}

Colorectal cancer (CRC) is one of the leading causes of cancer-related death worldwide ${ }^{1,2}$, with an estimated 1.8 million cases yearly and an annual mortality of over $881,000^{3}$. Due to the relatively asymptomatic progression of the disease in the early stages, patients are frequently diagnosed with metastatic disease, with a 5-year survival rate of around $14 \%{ }^{4}$. Current chemotherapies include 5-

\footnotetext{
Correspondence: Qiong Luo (qiongluo@nju.edu.cn) or

Qiang Xu (molpharm@163.com)

'State Key Laboratory of Pharmaceutical Biotechnology, Nanjing Drum Tower Hospital and School of Life Sciences, Nanjing University, 210023 Nanjing, China ${ }^{2}$ Department of Oncology, The First Affiliated Hospital with Nanjing Medical University, 210029 Nanjing, China

Full list of author information is available at the end of the article.

These authors contributed equally: Yunlong Shan, Yuan Gao

Edited by C. Munoz-Pinedo
}

flurouracil (5-FU), oxaliplatin and irinotecan. The monoclonal antibodies including vascular endothelial growth factor (VEGF) inhibitor bevacizumab and epidermal growth factor receptor (EGFR) inhibitor cetuximab are used along with 5-FU-based therapy for metastatic CRC. Despite these therapeutic advances, the latest adjuvant clinical trials have shown that the addition of targeted agents to standard chemotherapies has no value in advanced $\mathrm{CRC}^{5-8}$.

The essential hallmarks of cancer are intertwined with an altered cancer cell intrinsic metabolism ${ }^{9}$. Metabolic changes in tumor cells directly regulate development and progression of cancer. Cancer cells adjust corresponding energy metabolism in order to fuel cell growth and divi$\operatorname{sion}^{10}$. To meet the energy requirements of cancer cells, the entire metabolism, especially the tricarboxylic acid

\section{(c) The Author(s) 2019}

(c) (i) Open Access This article is licensed under a Creative Commons Attribution 4.0 International License, which permits use, sharing, adaptation, distribution and reproduction in any medium or format, as long as you give appropriate credit to the original author(s) and the source, provide a link to the Creative Commons license, and indicate if changes were made. The images or other third party material in this article are included in the article's Creative Commons license, unless indicated otherwise in a credit line to the material. If material is not included in the article's Creative Commons license and your intended use is not permitted by statutory regulation or exceeds the permitted use, you will need to obtain permission directly from the copyright holder. To view a copy of this license, visit http://creativecommons.org/licenses/by/4.0/. 
(TCA) cycle, is reorganized to augment anabolic reactions associated with cell growth and proliferation. Thus, metabolic reprogramming as a well-appreciated hallmark of cancer has been extensively used for drug discovery research $^{11,12}$.

Branched-chain amino acids (BCAAs) including leucine, isoleucine, and valine, are essential amino acids, and they have recently emerged as predictors for the future risk of cancers and diabetes ${ }^{13,14}$. Two different types of tumors, specifically pancreatic ductal adenocarcinoma and nonsmall-cell lung carcinoma, were recently shown to exhibit different usages of BCAAs ${ }^{15}$. 3-hydroxyisobutyryl-CoA hydrolase $(\mathrm{HIBCH})$, the enzyme which catalyzes the conversion of 3-hydroxyisobutyryl-CoA to 3hydroxyisobutyrate ${ }^{16}$, is a critical mitochondrial protein in valine catabolism ${ }^{17,18}$. The metabolite 3-hydroxyisobutyrate is further converted to succinyl-CoA and participates in metabolism of TCA cycle (Fig. S1). Mutations in the $\mathrm{HIBCH}$ gene are present at low levels $(0.5-6.0 \%)$ in many kinds of cancers. Moreover, it has been reported that $\mathrm{HIBCH}$ is increased in the patients with prostate cancer ${ }^{19}$. Thus, the critical roles of $\mathrm{HIBCH}$ in cancer cell growth and valine homeostasis suggest the potential for new therapies targeting valine metabolism by antagonizing $\mathrm{HIBCH}$. In this study, we demonstrated for the first time that $\mathrm{HIBCH}$ played an important role in the pathogenesis of CRC. We found that a synthetic steroidal glycoside SBF-1 inhibited TCA cycle and oxidative phosphorylation (OXPHOS) by blocking mitochondrial localization of $\mathrm{HIBCH}$, and thus showed its remarkable antitumor effects against CRC both in vitro and in vivo. Interestingly, SBF-1 was also able to overcome the resistance of CRC to the anti-VEGF therapy with bevacizumab by interfering mitochondrial $\mathrm{HIBCH}$, which can be used as a new combined therapy for CRC.

\section{Results \\ High HIBCH expression correlates with poor survival in patients with CRC}

How HIBCH influences CRC initiation and progression remains unknown. To explore the clinical relevance of $\mathrm{HIBCH}$ expression and CRC, we firstly analyzed the data from the Gene Expression Omnibus (GEO) datasets. Kaplan-Meier analysis showed that the increased $\mathrm{HIBCH}$ expression was positively related with a poor overall patient survival (Fig. 1a), and patients with higher $\mathrm{HIBCH}$ levels had a significantly shorter disease-free survival vs. patients with lower HIBCH levels (Fig. 1b). Then, $\mathrm{HIBCH}$ protein level was evaluated by immunohistochemical staining in 17 paired CRC tissues and the para-carcinoma tissues (Fig. 1c, d). We found that HIBCH expression significantly increased in the tumor tissues as compared with the para-carcinoma tissues (Fig. 1e and Fig. S2). These results suggest that high $\mathrm{HIBCH}$ expression may play an important role in the pathogenesis of CRC.

\section{HIBCH is involved in CRC cell growth and mitochondrial respiration}

To investigate the function of $\mathrm{HIBCH}$ in $\mathrm{CRC}$, we overexpressed and silenced HIBCH in HCT116 cells (a colorectal carcinoma cell line). As shown in Fig. 2a, b, silence of HIBCH inhibited the viability of HCT116 cells, whereas $\mathrm{HIBCH}$ overexpression promoted the cell viability. The EdU incorporation assay demonstrated that HIBCH silence resulted in an obvious decrease in counts of EdU incorporated cells (Fig. 2c). Instead, overexpression of $\mathrm{HIBCH}$ in HCT116 cells presented an increase in the percentage of EdU-positive cells in a timedependent manner (Fig. 2d). HIBCH is a nuclear-encoded mitochondrial enzyme with an $\mathrm{N}$-terminal transit peptide, which is required for localization to the mitochondrion (Fig. S3a). When the transit peptide was removed $(\triangle \mathrm{HIBCH})$, the cell proliferation promoted by $\mathrm{HIBCH}$ overexpression was diminished (Fig. 2e).

Flow cytometric analysis of cell apoptosis showed that HIBCH knockdown induced apoptosis of HCT116 cells (Fig. $2 \mathrm{f}$ and Fig. S3b). It is reported that autophagy is an alternative cell death-related pathway. Therefore, we examined the protein levels of LC3 and p62 (two widely used markers of autophagy) to determine whether $\mathrm{HIBCH}$ regulated autophagy of CRC. LC3 conversion (LC3-I to LC3-II) and p62 degradation were found in the HIBCH deficiency HCT116 cells, as compared with the control cells (Fig. 2g). Next, we analyzed the impact of HIBCH on AMP-activated protein kinase (AMPK), which is activated in response to nutrient- and energy-poor conditions ${ }^{20}$ and responsible for the initiation step of autophagy. As a result, silence of $\mathrm{HIBCH}$ increased AMPK phosphorylation, decreased mTOR phosphorylation, and concomitantly induced the activation of autophagy-initiating complex component $\mathrm{ULK}^{21}$. In particular, we found increased ULK1 phosphorylation levels at Ser555 (active ULK1 form) and decreased levels at Ser757 (inactive ULK1 form) (Fig. 2g and Fig. S3c). Overall, mitochondrial $\mathrm{HIBCH}$ is required for CRC cell growth.

Autophagy is often triggered by a cellular metabolic imbalance. Therefore, we determined whether there was an altered metabolic state caused by $\mathrm{HIBCH}$ deficiency or overexpression in HCT116 cells. Using ultrafast liquid chromatography-mass spectrometry (UFLC-MS) analysis, we found that the generation of 3hydroxyisobutyrate was decreased while excess 3hydroxyisobutyryl-CoA was detected by knockdown of $\mathrm{HIBCH}$, indicating that intracellular valine catabolism was altered in these cells. In contrast, $\mathrm{HIBCH}$ overexpression resulted in abundant 3-hydroxyisobutyrate (Fig. 3a and Fig. S4a). In valine catabolism, 3hydroxyisobutyrate is further converted to final product propionyl-CoA, and then to succinyl-CoA via a series of enzymatic reactions and participates in 

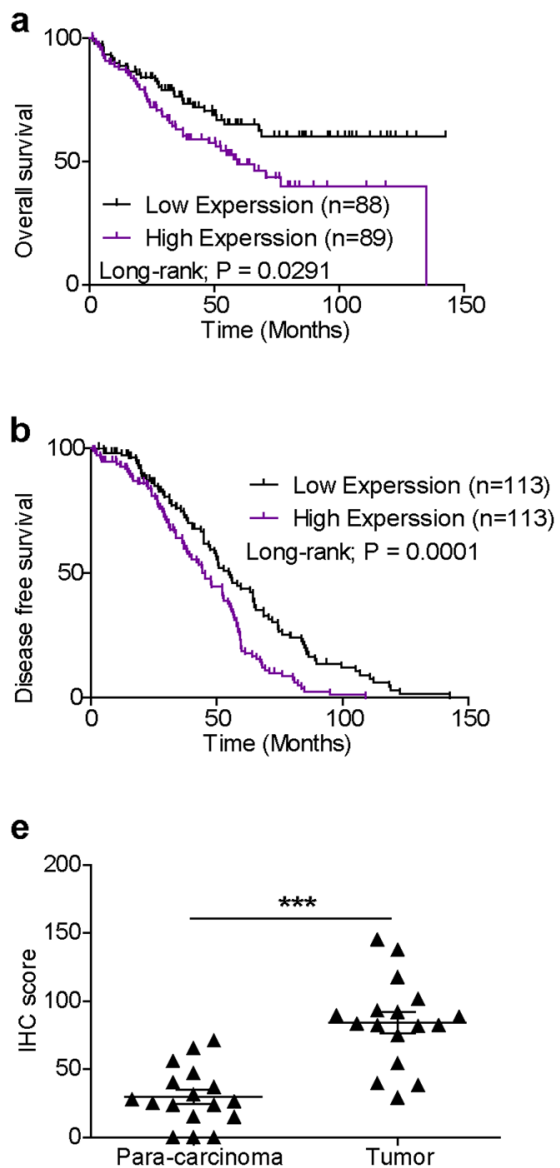

C
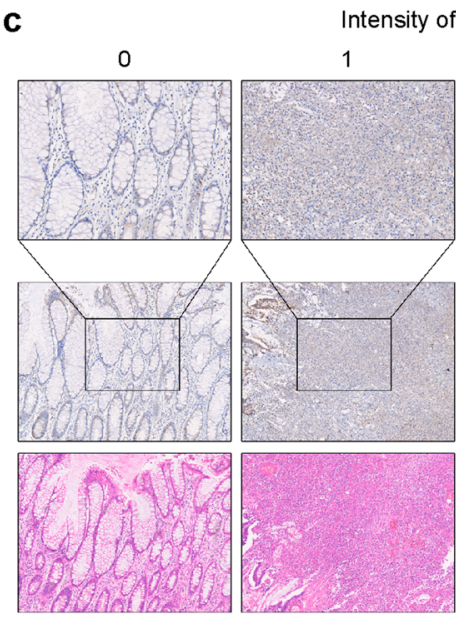

Intensity of IHC staining
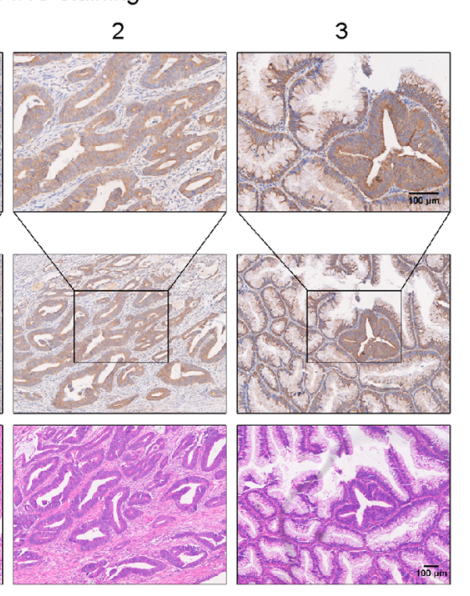

d

\begin{tabular}{|l|c|c|c|c|}
\hline Patient ID & $\begin{array}{c}\text { Prar-carcinoma } \\
\text { Tissues }\end{array}$ & Positive Area & Tumor & Positive Area \\
\hline 20120565 & 1 & 31.6 & 2 & 41.2 \\
\hline 20120648 & 2 & 18.5 & 3 & 39.2 \\
\hline 20120676 & 2 & 20.2 & 3 & 25.0 \\
\hline 20120692 & 1 & 23.9 & 2 & 41.3 \\
\hline 20120694 & 0 & 20.7 & 2 & 19.9 \\
\hline 20121043 & 1 & 26.6 & 2 & 46.1 \\
\hline 20121053 & 1 & 15.3 & 2 & 44.3 \\
\hline 20121071 & 0 & 18.9 & 3 & 48.4 \\
\hline 20121081 & 0 & 21.7 & 2 & 41.0 \\
\hline 20121216 & 1 & 25.3 & 3 & 45.9 \\
\hline 20181889 & 1 & 27.9 & 2 & 41.8 \\
\hline 20120637 & 1 & 23.5 & 1 & 38.4 \\
\hline 20120691 & 2 & 23.7 & 2 & 27.2 \\
\hline 20121147 & 1 & 15.1 & 1 & 29.3 \\
\hline 20121201 & 2 & 35.6 & 2 & 46.6 \\
\hline 20121290 & 3 & 18.7 & 3 & 34.0 \\
\hline 20120693 & 3 & 21.9 & 2 & 44.8 \\
\hline
\end{tabular}

Fig. 1 High HIBCH expression correlates with poor survival in patients with CRC. a HIBCH mRNA expression predicts poor survival in CRC patients in GSE17536. Patient survival data consisted of 177 CRC cases. b Cumulative DFS curves of HIBCH in GSE14333. Patient recurrence data consisted of 226 CRC cases. c Top and middle row, the protein level of HIBCH in tumors and para-carcinoma tissues from human CRCs was detected by immunohistochemistry (IHC); representative images of negative (intensity 0), weak (intensity 1), moderate (intensity 2), and strong (intensity 3 ) staining. Bottom row, H\&E staining. Scale bars, $100 \mu \mathrm{m}$. d Summary table of $\mathrm{IHC}$ staining area. e IHC scores of HIBCH in colorectal tumor and paracarcinoma tissues were calculated as follows: $\mathrm{HC}$ staining $\times$ positive area

metabolism of TCA cycle. We found that $\mathrm{HIBCH}$ knockdown resulted in decreased propionyl-CoA, while $\mathrm{HIBCH}$ overexpression greatly increased the levels of propionyl-CoA (Fig. S4b). We then found that $\mathrm{HIBCH}$ knockdown resulted in significant reduction in oxaloacetic acid and succinic acid (Fig. 3b), the metabolite of succinyl-CoA in TCA cycle. However, lack of $\mathrm{HIBCH}$ expression led to accumulation of fumarate and malic acid (Fig. 3b). It was reported that mitochondrial damage increased the level of fumarate during ische$\mathrm{mia}^{22}$. Previous studies also reported that $\mathrm{HIBCH}$ was important for modulating the toxic concentration of mitochondrial methacrylyl-CoA, which occurred in the middle part of valine catabolism and highly reacted with free thiol compounds, resulting in mitochondrial damage $^{23,24}$. It is likely that lack of HIBCH will cause mitochondrial damage, leading to the accumulation of fumarate and malic acid. These results suggested that HIBCH deficiency might affect the activity of TCA cycle in CRC cells.

To further determine the potential downstream pathway of $\mathrm{HIBCH}$, we performed gene set enrichment analysis (GSEA) for oncogenic signatures in Molecular Signature Database (MSigDB). GSEA of colorectal adenocarcinoma data set from TCGA showed that oxidative phosphorylation signature was enriched in the samples with high expression of $\mathrm{HIBCH}$ (Fig. 3c). We found that $\mathrm{HIBCH}$ overexpression increased intracellular $\mathrm{NADH}$ levels. In contrast, shRNA-mediated depletion of $\mathrm{HIBCH}$ resulted in significant reduction of intracellular $\mathrm{NAD}^{+}$ and NADH levels in cells (Fig. 3d). Next, we set out to determine the role of $\mathrm{HIBCH}$ in oxygen consumption. We compared the oxygen consumption rate (OCR) in the control HCT116 cells to that of transfected cells, and 


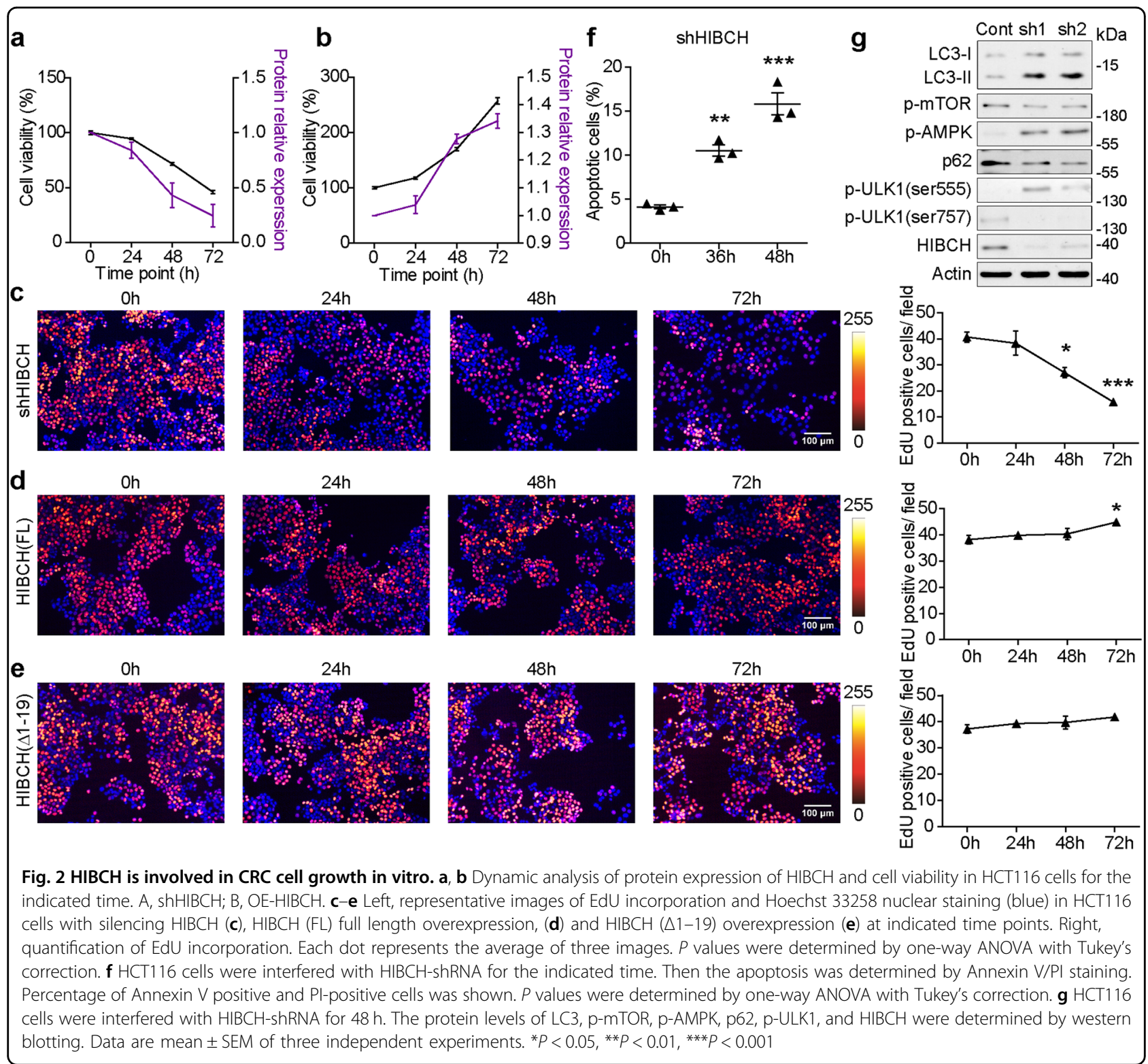

found that $\mathrm{HIBCH}$ knockdown decreased mitochondrial respiration, spare respiratory capacity, and ATP production, whereas HIBCH overexpression increased OCR (Fig. $3 \mathrm{e})$, thus indicating that $\mathrm{HIBCH}$ increased oxidative phosphorylation activity in CRC cells.

\section{Decreasing HIBCH mitochondrial localization by SBF-1 contributes to the inhibition of CRC cell growth}

To examine the possibility to target $\mathrm{HIBCH}$ for the treatment of $\mathrm{CRC}$, we screened some small molecule compounds on $\mathrm{HIBCH}$ expression and function and found that the synthetic steroidal glycoside SBF-1 showed a unique function against $\mathrm{HIBCH}$. Namely, it timedependently inhibited the localization of $\mathrm{HIBCH}$ to mitochondria without affecting total HIBCH expression by both immunoblot assay and immunofluorescence staining (Fig. 4a, b). Computational modeling of SBF-1$\mathrm{HIBCH}$ suggested that the side chain of SBF-1 impaired the recognition of TOM20 localized in the mitochondrial outer membrane (Fig. S5a). Then we measured binding of SBF-1 to GFP-HIBCH using microscale thermophoresis. Normalization and fitting of data from three independent measurements demonstrated that SBF-1 binds to $\mathrm{HIBCH}$ with a $K_{D}$ of $35.878 \mu \mathrm{M}$ (Fig. S5b). Considering the AKT and $\mathrm{Ca}^{2+}$ homeostasis inhibition of SBF-1, we investigated the effect of AKT inhibitor (MK2206) or ER $\mathrm{Ca}^{2+}$-ATPase inhibitor (thapsigargin, TG) on $\mathrm{HIBCH}$ localization. We found that MK2206 and TG failed to induce $\mathrm{HIBCH}$ 

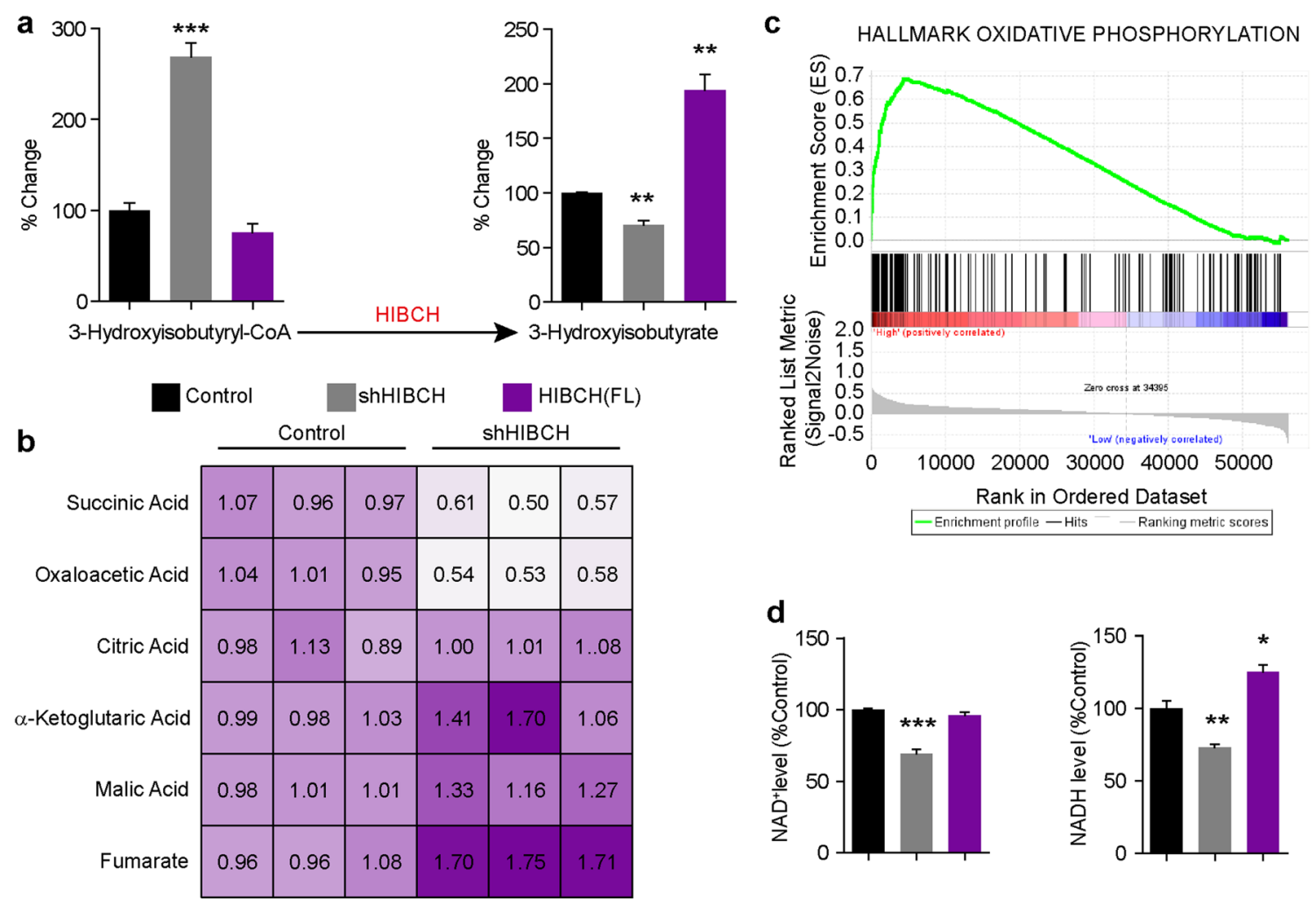

-Enrichment profile - Hits - - Ranking metric scores
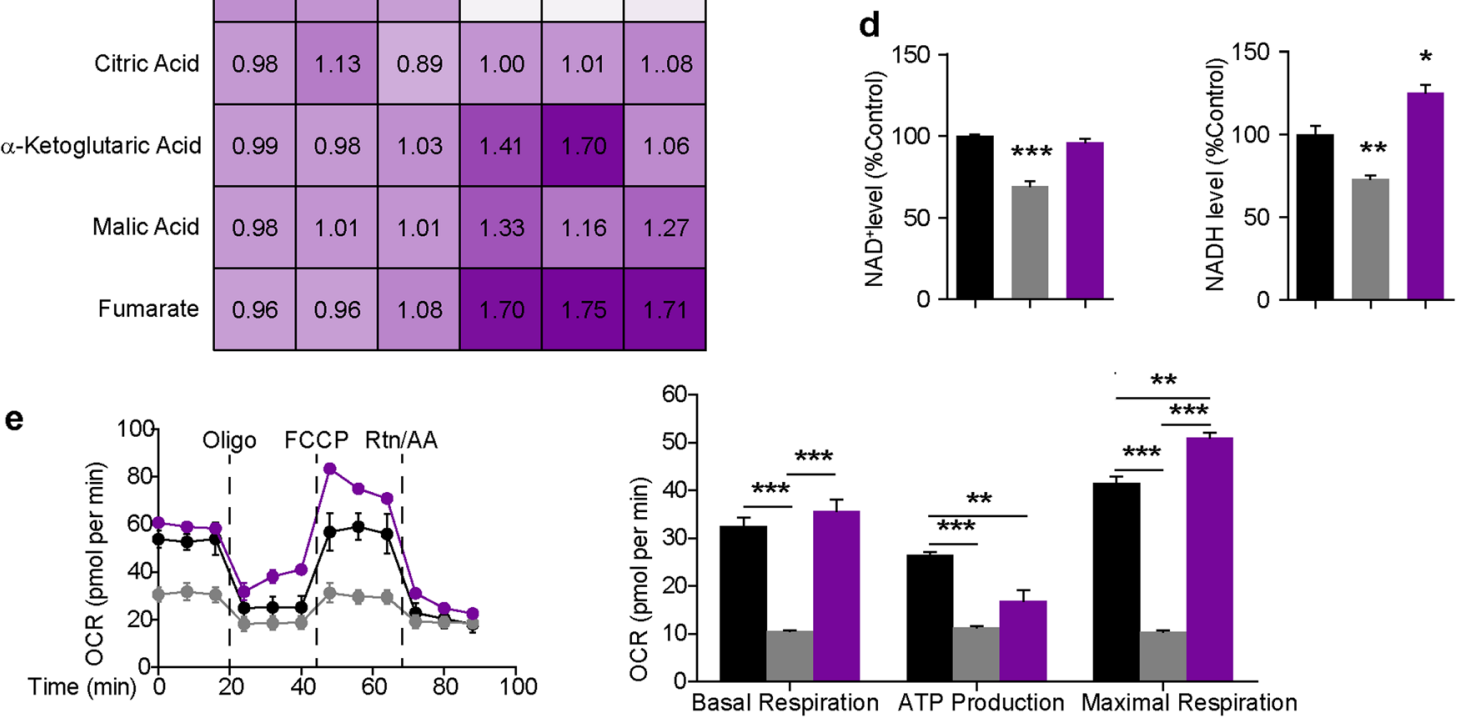

Fig. 3 HIBCH deficiency results in decreased TCA cycle and OXPHOS in CRC cells. HCT116 cells were overexpressed with HIBCH vector or interfered with HIBCH-shRNA for $48 \mathrm{~h}$. a 3-hydroxyisobutyryl-CoA (left) and 3-hydroxyisobutyrate (right) levels in cells were measured by UFLC-MS. $P$ values were determined by Student's $t$ test. $\mathbf{b}$ Metabolites of the TCA cycle in HCT116 cells were quantified by UFLC-MS as indicated treatment. $P$ values were determined by Student's $t$ test. c Gene set enrichment analysis using a gene set representing all genes significantly higher expressed $\mathrm{HIBCH}$ in CRC patients from TCGA compared with low expression, comparing cumulative expression levels from oxidative phosphorylation hallmark. d NAD ${ }^{+}$(left) and NADH (right) levels in HCT116 cells as indicated treatment were measured by EnVision multimode plate reader. $P$ values were determined by one-way ANOVA with Tukey's correction. e Right, the OCR in HCT116 cells in the presence of the indicated treatment was measured by Seahorse XF Analyzers; left, graph quantification of basal respiration, ATP production, and maximal respiration from each group. Data are mean \pm SEM of three independent experiments. ${ }^{*} P<0.05,{ }^{*} P<0.01$, ${ }^{* *} P<0.001$

mitochondrial translocation (Fig. S5c). SBF-1 inhibited the proliferation of HCT116 cells in a dose- and timedependent manner (Fig. 4c, d, Fig. S5d) and induced apoptosis in HCT116 cells (Fig. S5e). To further demonstrate the role of $\mathrm{HIBCH}$ in the antitumor effects of SBF1, we overexpressed $\mathrm{HIBCH}$ and then treated with SBF-1 in HCT116 cells. As shown in Fig. 4e, HIBCH overexpression rescued the reduction of EdU incorporation induced by SBF-1, whereas $\triangle \mathrm{HIBCH}$ did not have this effect (Fig. 4f). The above results revealed that SBF-1 decreased CRC cell proliferation by inhibiting $\mathrm{HIBCH}$ mitochondrial localization.

\section{SBF-1 reprograms both TCA cycle metabolism and oxidative phosphorylation dependent on $\mathrm{HIBCH}$ in CRC cells}

To address whether the change in $\mathrm{HIBCH}$ localization induced by SBF-1 has any effect on cellular metabolism, we first examined the effect of SBF-1 on valine catabolism. As shown in Fig. 5a and Fig. S4c, treatment 


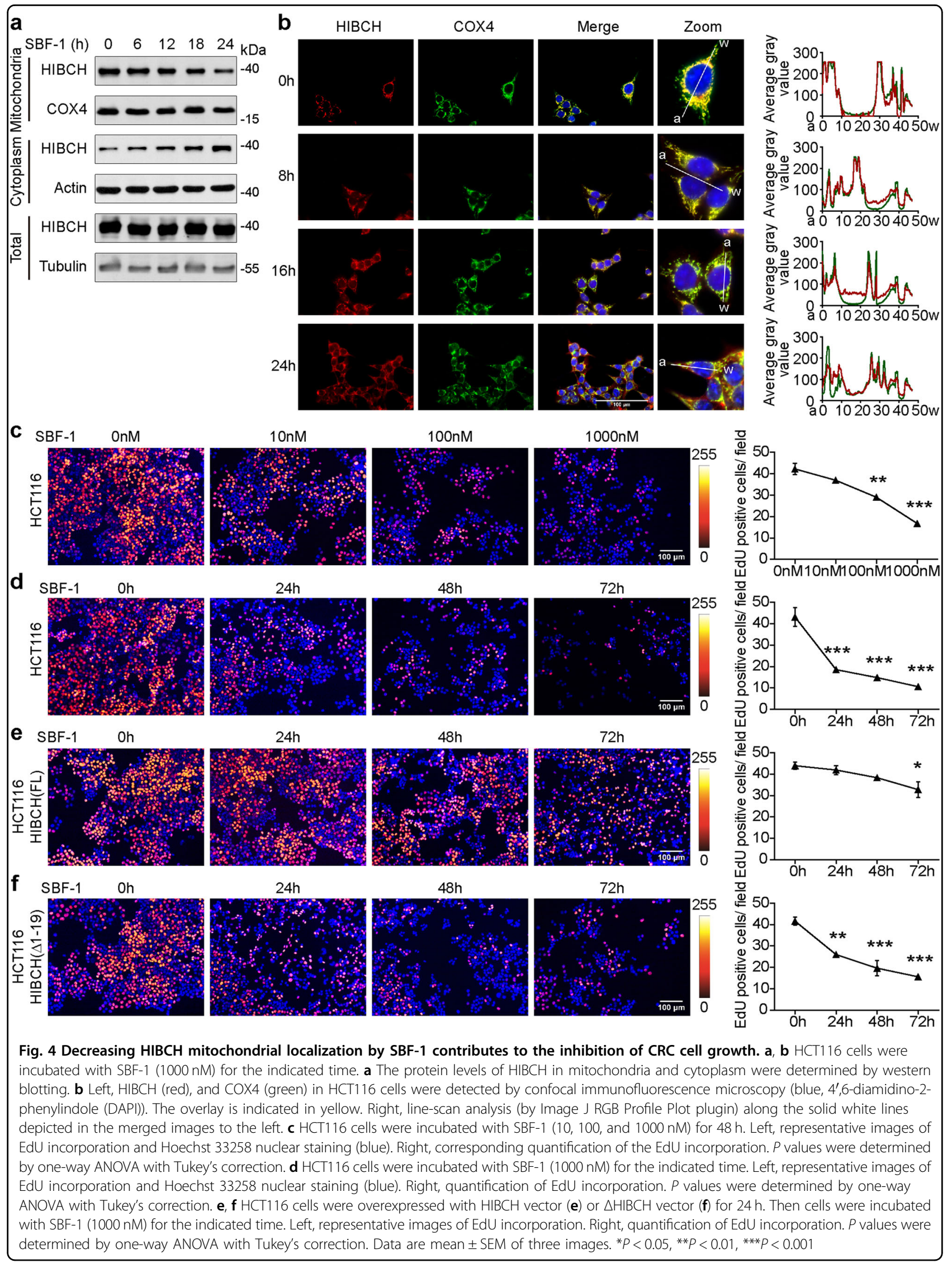



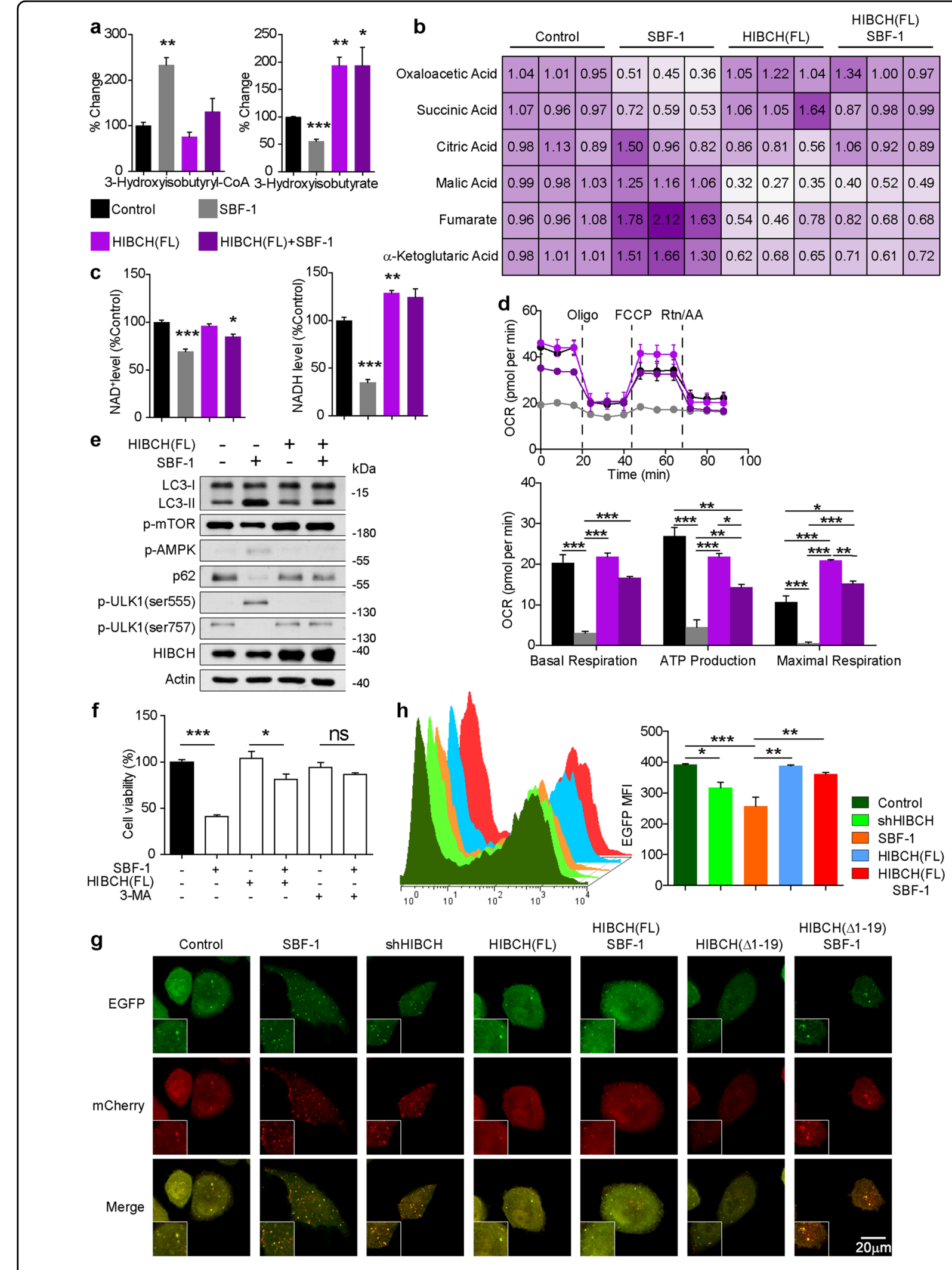

Fig. 5 (See legend on next page.) 


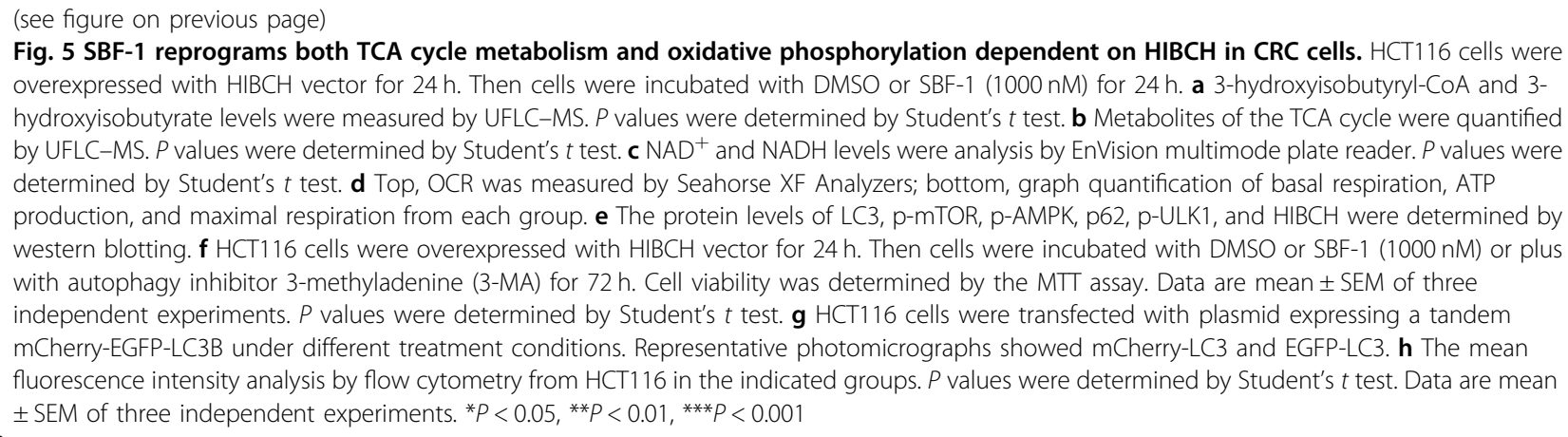

with SBF-1 (1000 nM) decreased the generation of 3hydroxyisobutyrate and propionyl-CoA, whereas 3hydroxyisobutyryl-CoA levels were increased. $\mathrm{HIBCH}$ overexpression rescued SBF-1-inhibited the hydrolysis of 3-hydroxyisobutyryl-CoA (Fig. 5a). Incubation of SBF-1 resulted in a significant decrease of succinic acid in TCA cycle, whereas overexpression of $\mathrm{HIBCH}$ increased the level of succinic acid in SBF-1-treated HCT116 cells (Fig. 5b). In addition, accumulation of fumarate and malic acid was also observed in SBF-1treated HCT116 cells (Fig. 5b). Furthermore, treatment with SBF-1 significantly attenuated $\mathrm{NAD}^{+}$and $\mathrm{NADH}$ levels (Fig. 5c) and the OCR (Fig. 5d). SBF-1-associated $\mathrm{NADH}$ reduction and OCR inhibition were also reversed by $\mathrm{HIBCH}$ overexpression (Fig. 5c, d), indicating that $\mathrm{HIBCH}$ was required for the suppression of oxidative phosphorylation by SBF-1 in HCT116 cells.

We then investigated the effects of SBF-1 on autophagy. As shown in Fig. 5e, LC3 conversion and p62 degradation were found in SBF-1-treated HCT116 cells, consistent with initiation of the metabolicsensing pathway of autophagy. In addition, SBF-1 increased AMPK phosphorylation, decreased mTOR phosphorylation, and concomitantly induced the activation of ULK1. In particular, increased ULK1 phosphorylation levels at Ser555 and decreased levels at Ser757 were confirmed. The above changes in autophagic parameters induced by SBF-1 were diminished or almost completely blocked by the overexpression of HIBCH (Fig. 5e) but not $\triangle$ HIBCH (Fig. S6). Besides, the effects of SBF-1 on cell viability were rescued by autophagy inhibitor 3-methyladenine and $\mathrm{HIBCH}$ overexpression (Fig. 5f). We further examined the expression of a tandem mCherry-EGFP-LC3 reporter under different treatment conditions in CRC cells. Because the EGFP fluorescence signal is quenched in acidified compartments, the immature autophagosomes are visible as yellow puncta, while red-only puncta represent acidified autolysosomal structures ${ }^{25}$. We here found that SBF-1 treatment or $\mathrm{HIBCH}$ knockdown (Fig. 5g) resulted in autophagy, which was characterized by the formation of red puncta (autolysosomal) in cytoplasm. Overexpression of $\mathrm{HIBCH}$ reduced the formation of red puncta, but this phenomenon was not observed in cells transfected with $\triangle \mathrm{HIBCH}$ (Fig. 5g). As expected, we validated the above results by flow cytometry (Fig. $5 \mathrm{~h}$ ). Thus, these data demonstrated the reduction of $\mathrm{HIBCH}$ mitochondrial localization inhibited valine catabolism, which in turn triggered autophagy in CRC cells.

\section{SBF-1 strongly inhibits the growth of human CRC xenografts in mice via blocking $\mathrm{HIBCH}$ mitochondrial localization}

To evaluate the antitumor effects of SBF-1 in vivo, human CRC-bearing nude mice were intraperitoneally injected with SBF-1 for treatment. Tumor volumes and body weights were measured every 2 days. The growth of tumor xenografts and tumor weights were significantly inhibited by SBF-1 at the very low dose of 1 and $5 \mu \mathrm{g} / \mathrm{kg}$ in both HT-29 (Fig. 6a, c and Fig. S7a) and HCT116 (Fig. 6b) xenograft models. In mice bearing HT-29 or HCT116 CRC, only $5 \mu \mathrm{g} / \mathrm{kg}$ of SBF- 1 slightly reduced weight gain (Fig. S7b and c). As compared with vehicle group, Kaplan-Meier analysis showed that SBF1 significantly prolonged the overall survival of the tumor-bearing mice inoculated with HT-29 cells (median (50\%) survival: vehicle, 63 days; SBF-1 $1 \mu \mathrm{g} / \mathrm{kg}$, 72 days; SBF-1 $5 \mu \mathrm{g} / \mathrm{kg}, 74$ days) (Fig. $6 \mathrm{~d}$ ). In this case, LC3 conversion (LC3-I to LC3-II) and p62 degradation in tumors were observed due to the SBF-1 treatment (Fig. 6e). Similarly, immunofluorescence staining of p62 showed a significant decrease in p62-positive cells in SBF-1-treated tumors compared with the vehicle group (Fig. 6f). Furthermore, SBF-1 treatment reduced $\mathrm{HIBCH}$ expression in mitochondria in tumor tissues compared with vehicle-treated xenografts (Fig. 6g). SBF-1 also significantly inhibited the expression of proliferating cell nuclear antigen (PCNA) (Fig. 6h). In contrast, terminal deoxynucleotidyl transferase dUTP 


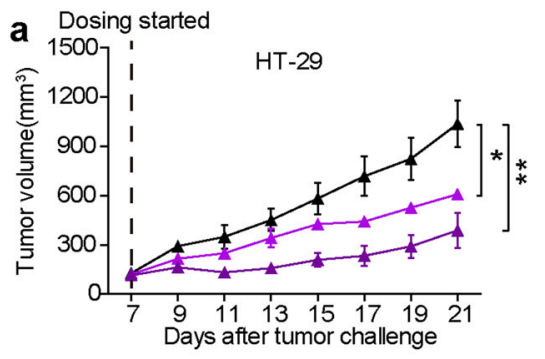

d

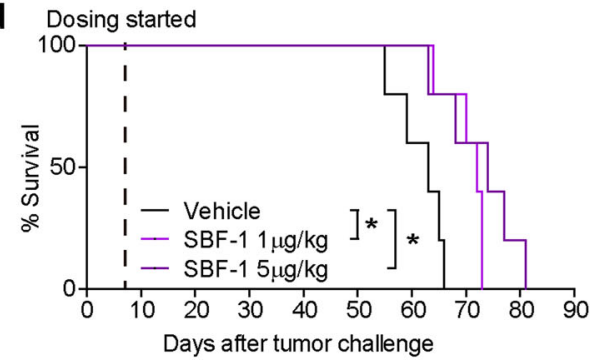

e

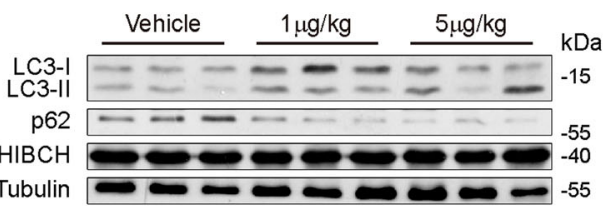

h
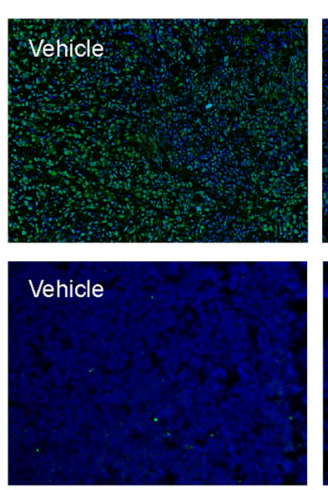

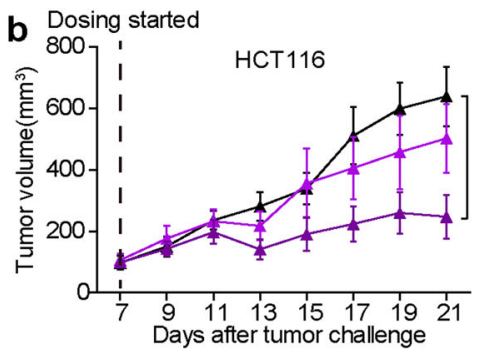

f

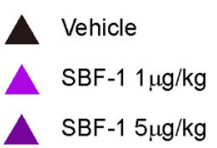

$\mathrm{SBF}-15 \mu \mathrm{g} / \mathrm{kg}$
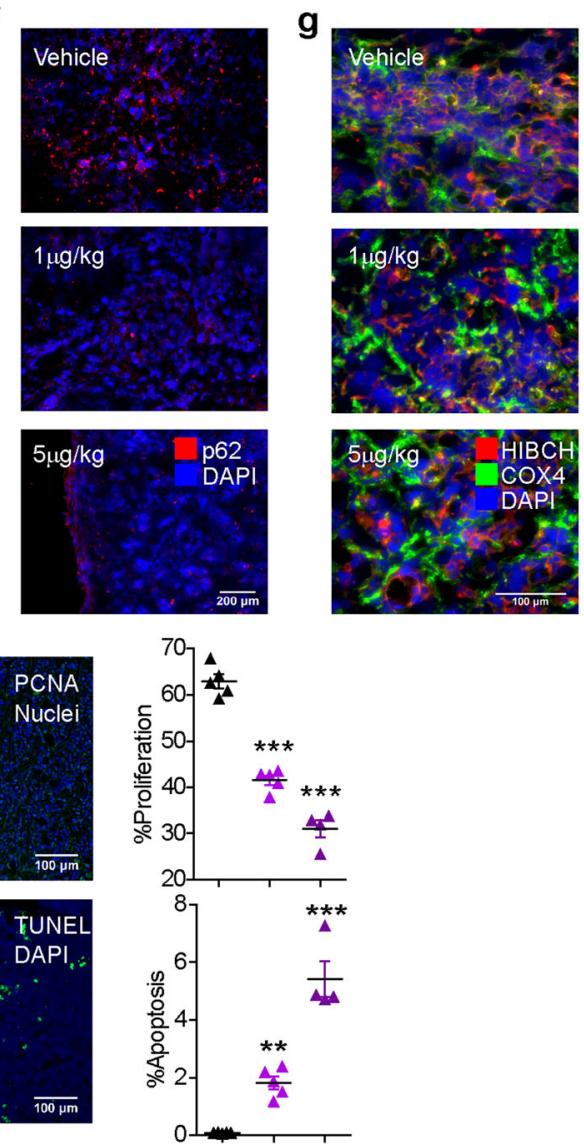

Fig. 6 SBF-1 strongly inhibits the growth of human CRC xenografts in mice via blocking HIBCH mitochondrial localization. a, b Volumetric analysis over a 21-days treatment regimen (with vehicle or 1, $5 \mu \mathrm{g} / \mathrm{kg} \mathrm{SBF}-1$ for 2 weeks following a $5 \mathrm{on} / 2$ off dosing schedule) in athymic nude mice with HT-29 a and HCT116 b cell line xenografts. $n=5$ mice per group. $P$ values were determined by Student's $t$ test. $\mathbf{c}$ Tumor weights of HT-29 xenografts at necropsy. Each dot represents one tumor. $n=5, P$ values were determined by Student's $t$ test. $\mathbf{d}$ Kaplan-Meier survival analysis of mice subcutaneous implanted with HCT116 cells and treated with vehicle or with 1 and $5 \mu \mathrm{g} / \mathrm{kg} \mathrm{SBF-1.} n=5$. Statistical analysis is determined by log-rank test. e The protein levels of $\mathrm{LC} 3, \mathrm{p} 62$, and $\mathrm{HIBCH}$ in tumor samples from the indicated groups were determined by western blotting. $\mathbf{f} \mathrm{P} 62$ in xenograft tumors from the indicated groups was detected by immunofluorescence microscopy. $\mathbf{g} \mathrm{HIBCH}$ and COX4 in xenograft tumors in the indicated groups were detected by immunofluorescence microscopy. $\mathbf{h}$ Left, representative images of PCNA immunostaining and nuclear staining of tumors in different treatment groups. Right, relative nuclear PCNA of the indicated groups. Each symbol represents the mean of five random fields from a single mouse. Number of experimental mice: vehicle, $n=5 ;$ SBF-1 $1 \mu \mathrm{g} / \mathrm{kg}, n=5 ;$ SBF-1 $5 \mu \mathrm{g} / \mathrm{kg}, n=4, P$ values were determined by one-way ANOVA with Tukey's correction. i Left, representative images of TUNEL and DAPI of tumors in different treatment groups. Right, relative apoptosis of the indicated groups. Each symbol represents the mean of five random fields from a single mouse. Number of experimental mice are same in h. $P$ values were determined by one-way ANOVA with Tukey's correction. Error bars represent \pm SEM. ${ }^{*} P<0.05$, ${ }^{* *} P<0.01,{ }^{* * *} P<0.001$

nick-end labeling positive cells were significantly increased in tumors of SBF-1 treated mice (Fig. 6i). Taken together, the in vivo study strongly suggested that blocking mitochondrial localization of $\mathrm{HIBCH}$ by SBF-1 could be effective in inhibiting tumor growth in CRC xenograft models. 
HIBCH links between valine catabolism and anti-VEGF therapy and targeting $\mathrm{HIBCH}$ overcomes the bevacizumab resistance

Notwithstanding the clinical use of bevacizumab with chemotherapy for CRCs, many patients are refractory to bevacizumab ${ }^{26}$, and biomarkers to identify responders are missing ${ }^{27}$. In a recent trial, bevacizumab prolonged disease-free progression but not the overall survival ${ }^{28}$, and failed to show benefit in the adjuvant setting ${ }^{29}$. Previous studies showed that treatment with bevacizumab altered cellular metabolism in CRC cells. The upregulation of HIBCH in HT-29 tumor xenograft treated with bevacizumab was observed by global proteomic profiling ${ }^{30}$. This finding hints a possibility that the upregulated $\mathrm{HIBCH}$ may restrict the clinical efficacy of bevacizumab. Indeed, the present study demonstrated that treatment with bevacizumab elevated both protein and mRNA levels of $\mathrm{HIBCH}$ in CRC cells in time- and dose-dependent manners (Fig. 7a, b), which may be back to bite the effect of the drug. As expected, high $\mathrm{HIBCH}$ expression greatly induced bevacizumab resistance in HCT116 cells (Fig. S2d). To overcome this issue in bevacizumab, we assessed the in vivo therapeutic potential of SBF-1 and bevacizumab combination and established a protocol in the HT-29 tumor xenograft model for the combined treatment (Fig. 7c). As the result, SBF-1 as low as $1 \mu \mathrm{g} / \mathrm{kg}$ significantly increased the antitumor effects of bevacizumab (Fig. 7d) and prolonged the overall survival of tumor-bearing mice (Fig. 7e) without affecting the body weights of mice. Together, our findings provided potential benefits for the application of bevacizumab in CRC via combination with SBF-1.

\section{Discussion}

The diversity of metabolic pathways that cancer cells rely on presents a key opportunity for drug development and precision medicine ${ }^{31}$. However, such diversity has also limited impact on the clinical management of cancer. This situation may partially arise from the fact that energy production in cancer cells is uniquely dependent on specific nutrients, such as BCAAs ${ }^{13}$.

The present study first found that upregulation of $\mathrm{HIBCH}$ occurred in CRC patients, which was associated with poor prognosis. Then we found that inhibition of $\mathrm{HIBCH}$ mitochondrial localization significantly reduced succinic acid in TCA cycle, decreased NADH and OCR,
A
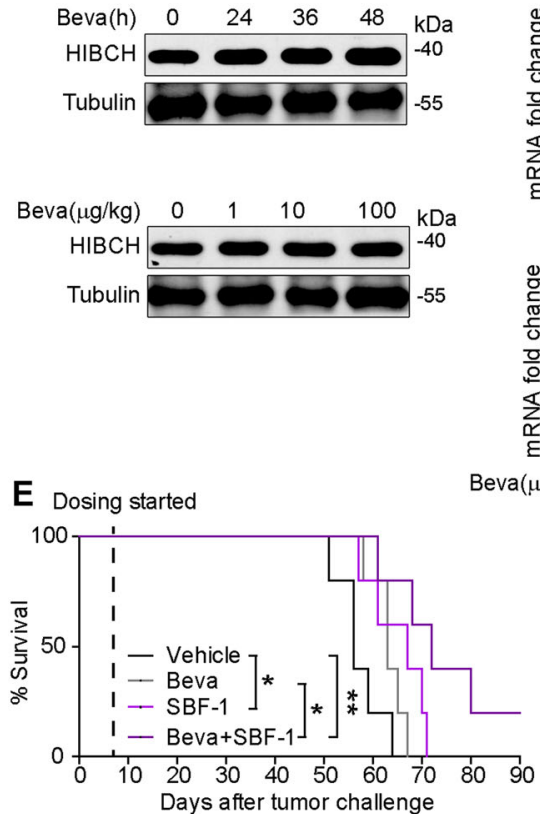
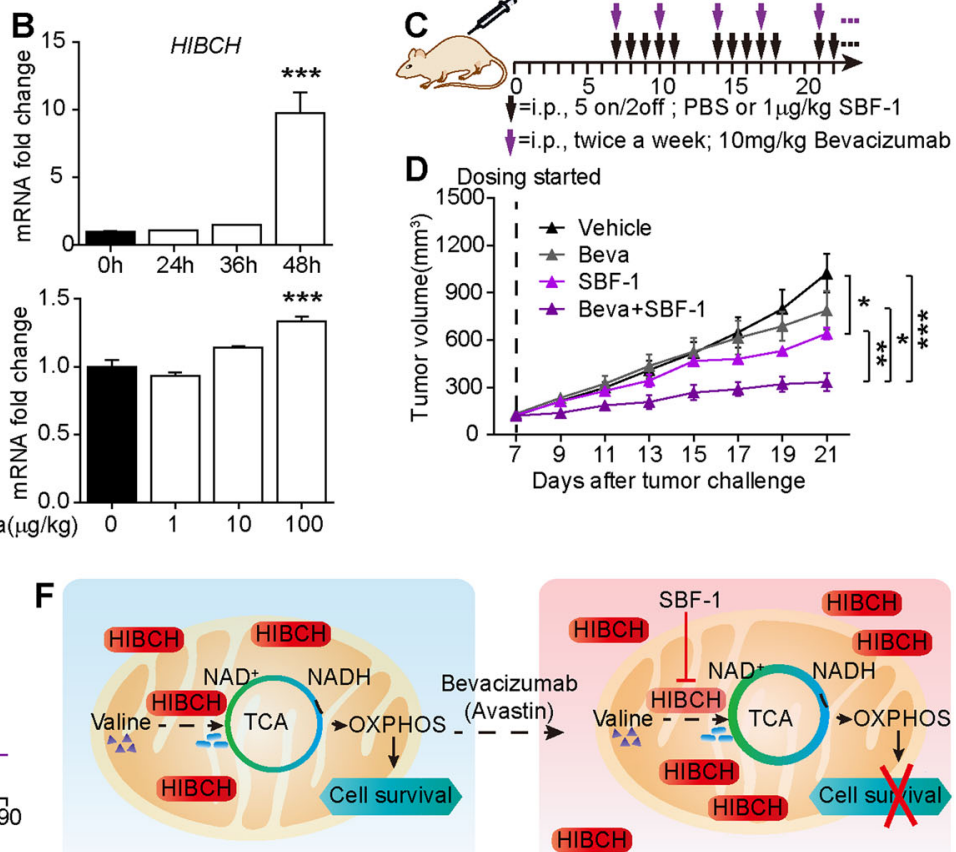

Fig. 7 SBF-1 overcomes the bevacizumab-induced high HIBCH for the combined therapy against CRC in preclinical models. $\mathbf{a}$, $\mathbf{b} H T-29$ cells were treated with bevacizumab in time- (top, $100 \mathrm{\mu g} / \mathrm{ml}$ for the indicated time) or dose-dependent manner (bottom, 1-100 $\mu \mathrm{g} / \mathrm{ml}, 48 \mathrm{~h}$ ). The protein levels of HIBCH were determined by western blotting (a) and mRNA levels of HIBCH were determined by real-time qPCR (b). Data are mean \pm SEM of three independent experiments. $P$ values were determined by one-way ANOVA with Tukey's correction. c Schematic of workflow for treatment as indicated. $\mathbf{d}$ Tumor volume of subcutaneous HT-29 xenograft tumors in mice from the indicated groups. $n=5, P$ values were determined by Student's $t$ test. e Kaplan-Meier survival analysis of mice subcutaneous implanted with HT-29 cells in the indicated groups. Statistical analysis by logrank test. $\mathbf{f}$ Model showing valine catabolism utilization by the TCA cycle and OXPHOS for cells survival, which are suppressed by SBF-1 via inhibition of $\mathrm{HIBCH}$ mitochondrial localization. Error bars represent \pm SEM. ${ }^{*} P<0.05,{ }^{* *} P<0.01,{ }^{* * *} P<0.001$ 
and concomitantly induced apoptosis and autophagy in CRC cells. Based on the evidence available, although the effect of lessened valine catabolism on $\mathrm{NAD}^{+}$and NADH consuming pathways warrants further investigation, we believed that $\mathrm{HIBCH}$ played an important role in mitochondrial TCA cycle and OXPHOS. This could be attributed to an accumulation of toxic valine metabolites. Besides, the current study discovered that knockdown of $\mathrm{HIBCH}$ facilitated initiation of autophagy and inhibited proliferation in CRC cells. By transfecting with $\mathrm{HIBCH}-$ shRNA, LC3 conversion (LC3-I to LC3-II) and p62 degradation were observed in HCT116 cells. HIBCH deficiency resulted in a metabolic crisis, during which the energy sensor kinase AMPK was activated and then responsible for autophagy initiation. However, more indepth investigations are required to elucidate the role of valine metabolism in CRC progression.

Another intriguing finding of this study was that a synthetic steroidal glycoside SBF-1 effectively inhibited $\mathrm{HIBCH}$ mitochondrial localization in CRC cells. We here identified SBF-1 as a first potent small molecule antagonist of valine catabolism. The metabolites from valine, which turn into TCA cycle, NADH levels, and OCR were all decreased by SBF-1. Elevated autophagy was another notable characteristic of the SBF-1-mediated response, which was consistent with diminished amino acid transport and metabolism ${ }^{32}$. SBF-1 increased AMPK phosphorylation, decreased the mTOR phosphorylation, and induced LC3-II activation and p62 degradation. Combination of SBF-1 with an autophagy inhibitor further rescued the viability of cancer cells, as elevated autophagy was likely a major response to SBF-1, potentially illuminating a future therapeutic strategy. The SBF-1-associated OCR inhibition and autophagy induction were reversed by $\mathrm{HIBCH}$ overexpression, but not $\triangle \mathrm{HIBCH}$, further indicating that mitochondrial localization of $\mathrm{HIBCH}$ was required for the suppression of cell growth by SBF-1 in HCT116 cells. Combined with these data, we supposed that SBF-1 might disrupt HIBCH mitochondrial localization and caused an apparent loss of HIBCH function in HCT116 cells, resulting in growth inhibition, which might explain the strong antitumor effect of SBF-1. Furthermore, in vivo studies revealed that both 1 and $5 \mu \mathrm{g} / \mathrm{kg}$ of SBF-1 significantly inhibited tumor growth and prolonged the overall survival of the tumor-bearing mice. Treatment with SBF-1 could promote autophagy through deceasing mitochondrial $\mathrm{HIBCH}$ localization. Importantly, the quantity and activity of $\mathrm{HIBCH}$ may represent a translational biomarker reflective of tumors likely to respond to SBF-1 and similar agents. Our study may also open opportunities for valine catabolism inhibitors for CRC therapy. Previous studies reported that $\mathrm{HIBCH}$ was critical for normal human development. Its mutations or deficiency inborn might lead to accumulation of toxic valine metabolites in mitochondria and cause Leigh syndrome or Leigh-like disease with mitochondrial disorders $^{23,24,33,34}$. Given that $\mathrm{HIBCH}$ is overexpressed in CRC tissues, CRC cells may be more sensitive to HIBCHtargeted therapy than normal cells. And low valine diet in CRC patients will help alleviate the side effects of methacrylyl-CoA on mitochondrial damage.

Antiangiogenesis with chemotherapy did not confer a survival benefit in disease confined to lymph nodes (stage III), and the optimal use of this modality remains to be defined $^{35}$. It is critical to unravel the mechanisms that underlie the resistance of colon cancer cells to antiangiogenic therapy. Targeting dysregulated signal transduction can be efficacious anticancer therapies with minimal adverse effects ${ }^{36}$. It has been demonstrated that anti-VEGF therapy with bevacizumab alters proteins involved in cellular metabolism on cancer cells, including HIBCH. Similar to David W. Greening's results ${ }^{27}$, our findings pointed out the possible implications of $\mathrm{HIBCH}$ upregulation by bevacizumab in CRC, although further evidence is needed. Thus, interference with $\mathrm{HIBCH}$ function may improve the efficacy of VEGF inhibition and lead to durable antitumor responses. Strikingly, we found that combination therapy with SBF-1 and bevacizumab significantly inhibited tumor growth in CRC models, leading to a robust survival benefit. Accordingly, colorectal cancer patients treated with bevacizumab could receive combinational use of valine metabolic inhibitors. Exploration of the therapeutic opportunities of bevacizumab and SBF-1 in CRC could be promising.

BCAAs, essential amino acids promoted protein synthesis and utilization in cancer patients ${ }^{37}$. Leucine and isoleucine metabolism to be an important "module" within cancer metabolism, which lead to glutamate formation for biosynthesis in cancer progression ${ }^{15,38,39}$. Previous studies have demonstrated total BCAAs were elevated in plasma of patients with pancreatic cancers, which can be converted to acetyl-CoA and enter the TCA cycle $^{40,41}$. However, the role of BCAAs metabolic inhibition in the cancer patients remains to be clearly defined. Branched-chain aminotransferase 1 (BCAT1), which converts BCAAs to the corresponding branched-chain $\alpha$ keto acids ${ }^{42,43}$, has been implicated in cancer growth ${ }^{44-47}$. Previously, Hattori et al. reported that altered BCAAs metabolism drove cancer progression in myeloid leuke$\mathrm{mia}^{13}$. BCAT1 enhances autophagy to induce chemoresistance $^{48}$ in hepatocellular carcinoma cells and promote mTOR activation in breast cancer ${ }^{47}$. In contrast, we found that silence of HIBCH increased AMPK phosphorylation, decreased mTOR phosphorylation, and concomitantly induced autophagy in CRC. Although BCAT1 is required for tumor growth and progression in a wide range of malignancies, BCAT1 inhibition impairs all three BCAAs metabolism. Given that $\mathrm{HIBCH}$ is only involved in valine 
catabolism and bevacizumab treatment increases $\mathrm{HIBCH}$ protein levels in $\mathrm{CRC}$, targeting $\mathrm{HIBCH}$ may be more specific and effective.

In conclusion, as illustrated in Fig. 7f, we demonstrated a key role of $\mathrm{HIBCH}$ in linking valine catabolism, TCA cycle and OXPHOS with CRC growth and found a first antagonist of $\mathrm{HIBCH}$ SBF-1. When CRC cells were exposed to SBF-1, the mitochondrial localization of $\mathrm{HIBCH}$ was suppressed, which resulted in a disturbance of valine catabolism, TCA cycle, and mitochondrial OXPHOS, activated autophagy and caused cell death. This study suggested that HIBCH could be a novel therapeutic target for CRC. Furthermore, since high $\mathrm{HIBCH}$ level was revealed as a self-induced reason of CRC to resist to anti-VEGF therapy, our findings also highlight a possibility of combination therapy using valine catabolic inhibitor along with anti-VEGF drugs, to control progression of advanced CRC.

\section{Materials and methods Reagents}

SBF-1 was synthesized by B. Y., a coauthor, as previously reported ${ }^{49}$. For in vitro experiments, SBF-1 was dissolved in DMSO to a concentration of $20 \mathrm{mM}$ (stock solution); and for the in vivo assay, $\mathrm{SBF}-1$ was dissolved in DMSO to a concentration of $1 \mathrm{mg} / \mathrm{ml}$ (stock solution). Bevacizumab (Avastin, Roche Diagnostics GmbH, Reinach, Switzerland) was diluted in phosphate buffer solution before use, and injected i.p. at the dose of $10 \mathrm{mg} / \mathrm{kg}$ twice a week.

\section{Immunohistochemical staining}

For IHC analysis, samples were collected and paraformaldehyde fixed, paraffin-embedded sections of tumor tissues $(5 \mu \mathrm{m}$ thick) were mounted on slides coated with 2-aminopropyltriethoxysilane, which then were baked, deparaffinized, rinsed with $3 \%$ hydrogen peroxide. After that, these sections were washed and then blocked with $3 \%$ goat serum for $5 \mathrm{~min}$ and subsequently incubated with an anti-HIBCH (Abcam, ab153826, Cambridge, UK; diluted 1:500) or PCNA (Santa Cruz Biotechnology, Santa Cruz, CA, USA, sc-56; diluted 1:100) antibody at at $4{ }^{\circ} \mathrm{C}$ overnight. Finally, the following steps were in accordance to the protocols provided by the manufacturer of GTVisionTMIII Complex (Gene Tech, GK500705, Shanghai, China). The nucleus was stained with hematoxylin (Solarbio, G1080, Beijing, China). Sections were further mounted with neutral gums. IHC sections were photographed by Mantra 1.01 (Perkin Elmer, Waltham, MA, USA).

\section{Cell lines and plasmid transfection}

Human colorectal cancer cells HCT116 and HT-29 were obtained from the Shanghai Institute of Cell Biology
(Shanghai, China) and were maintained in DMEM supplemented with $10 \%$ FBS plus $2 \mathrm{mM}$ glutamine, $100 \mathrm{U} / \mathrm{ml}$ penicillin, and $100 \mathrm{mg} / \mathrm{ml}$ streptomycin. Transient transfection of HCT116 cells were transfected with $2 \mu \mathrm{g}$ of $\mathrm{HIBCH}$ plasmid or shHIBCH (sh1: CCATACAGAGTCTAAGATT; sh2: GGTTACTTCCTTGCATTAA) using the Lipofectamine ${ }^{\circledast} 3000$ (Invitrogen, Carlsbad, CA, USA) reagents by following the manufacturer's instructions.

\section{EdU incorporation assay}

HCT116 were grown in 96-well plates and transfected with scramble or shHIBCH. Cells were collected at indicated time and stained with kFluor488 EdU Kit (KeyGEN Biotech, KGA331-500, Jiangsu, China) according to the manufacturer's instructions.

\section{Metabolite profiling of cultured cells}

Two million cells were washed three times with cold PBS and pelleted by centrifugation. Cell pellets were then flash frozen in liquid nitrogen and stored at $-80^{\circ} \mathrm{C}$ until further processing. Metabolites were extracted by adding $1 \mathrm{ml}$ of buffer (75\% of 9:1 methanol chloroform in HPLCgrade $\mathrm{H}_{2} \mathrm{O}$ ) to the cell pellets. The lysates were incubated at $4{ }^{\circ} \mathrm{C}$ for $20 \mathrm{~min}$ with shaking and cleared by centrifugation at $16,800 \mathrm{~g}$ for $5 \mathrm{~min}$ at $4{ }^{\circ} \mathrm{C}$. Two aliquots of supernatant were transferred to new Eppendorf tubes containing an acetonitrile/methanol (75/25, v/v) mixture. The samples were then dried-down using a SpeedVac and reconstituted with $100 \mu \mathrm{l}$ of $50 \%$ acetonitrile in water for the analysis.

Profiling of polar metabolites was performed by UFLC-MS in positive ion mode. Profiling of TCA cycle intermediates was performed by UFLC-MS in negative ion mode as previously described ${ }^{50,51}$. In brief, $10 \mu$ of reconstituted sample was loaded onto either an Xbrige C18 column $(100 \times 4.6 \mathrm{~mm}, 3.5 \mu \mathrm{m}$; Waters, Milford, MA, USA) for analysis. The metabolites were separated using a UFLC 20ADXR LC system in-line (Shimadzu Corporation, Kyoto, Japan) coupled with hybrid quadrupole timeof-flight tandem mass spectrometer (TripleTOFTM 5600 MS system, AB SCIEX Corporation, Toronto, Canada). MultiQuant software v2.1 (ABSCIEX) was used for automated peak integration and metabolite peaks were also assessed manually for quality of peak integration. UFLC-MS peak integration data of each sample were normalized to DNA content determined from a duplicate cell pellet.

\section{Oxygen consumption assay}

Adherent HCT116 cells were seeded at $5 \times 10^{3}$ cells/ well in $200 \mu \mathrm{l}$ of their culture medium and incubated for $24 \mathrm{~h}$ at $37^{\circ} \mathrm{C}$ in humidified atmosphere with $5 \% \mathrm{CO}_{2}$. After $1 \mathrm{~h}$ of incubation in a non- $\mathrm{CO}_{2}$ incubator, OCR was 
measured using the Seahorse Bioscience XF96 Extracellular Flux Analyzer (Seahorse Bioscience, CA, USA) where cells were subjected in sequence to the following additions: (1) basal levels were measured with no additives; (2) $1 \mu \mathrm{M}$ oligo, which reversibly inhibits ATP synthase and OXPHOS, was added to show ATP production; (3) $0.3 \mu \mathrm{M}$ FCCP, a mitochondrial uncoupler, was added to induce maximal respiration; (4) $0.1 \mu \mathrm{M}$ rotenone/ antimycin $\mathrm{A}$ was added to end the reaction. For the kinetics experiments, measurements were collected every $8 \mathrm{~min}$. Basal respiration was calculated as follows: last rate measurement before first injection-nonmitochondrial respiration rate. ATP production was calculated as follows: last rate measurement before oligomycin injection - minimum rate measurement after oligomycin injection. Maximal respiration was calculated as follows: first rate measurement after FCCP injection-nonmitochondrial respiration rate. The last rate measurement after rotenone/antimycin injection standed for nonmitochondrial respiration rate $^{52,53}$.

\section{Immunofluorescence (IF) staining}

The samples were permeabilized in $0.1 \%$ Triton X-100 and incubated with $1 \%$ BSA/PBS to block nonspecific binding. Subsequently, the cells were immunostained by incubating with $\mathrm{HIBCH}$ antibody (Abcam, ab153826; diluted 1:100), COX4 antibody (Santa Cruz Biotechnology, sc-376731; diluted 1:100), and p62 (diluted 1:100) overnight at $4{ }^{\circ} \mathrm{C}$. After being washed with PBS, cells were incubated with goat anti-rabbit AF594 (Thermo Fisher Scientific, Waltham, MA, USA, A-11037; diluted 1:500); donkey anti-mouse AF488 (Thermo Fisher Scientific, R37114; diluted 1:500). And nuclei were counterstained with DAPI (Beyotime, Shanghai, China; C1006). Fluorescent images were taken and analyzed using the ZEN pro 2012 imaging software on a Zeiss invert microscope under 100-630-fold magnification.

\section{Image analysis}

Line-scan analysis was performed using "RGB Profiler Plot" plugin as described previously ${ }^{54}$. Taken briefly, raw confocal images were background corrected. A straight line with "2" width was drawn in regions of interest. Then the lines were directly analyzed by choosing Image $\mathrm{J}$ plugin Graphics's "RGB Profiler Plot" under the "Plugins" menu. In the output of "RGB Profiler", data were exported into Microsoft Excel. The length of line was plotted on the $x$-axis, while the intensity was plotted on the $y$-axis.

\section{Mouse experiment}

Female BALB/c nude mice (6-8 weeks old, $18-20 \mathrm{~g}$ ) were purchased from the Model Animal Genetics Research Center of Nanjing University (Nanjing, China). HCT116 and HT-29 tumors were generated by injection of $1 \times 10^{7}$ cancer cells subcutaneously into the right flank of nude mice. The tumors were grown for several weeks, either according to a "survival" schedule (endpoint defined by tumor volume) or a fixed timepoint (endpoint defined as fixed time lapsed after tumor challenge). Tumor size was determined by caliper measurements and calculated using the formula: $V=0.5 \times\left[L \times S^{2}\right]$, where $S$ is the shorter and $L$ is the longer tumor axis. Tumor weight measurements were performed postmortem. All efforts were made to minimize the animals' suffering and to reduce the number of animals used. The medical ethical committee of Nanjing University approved all described studies.

\section{Preclinical mouse trials and drug regimens}

Vehicle (0.1\% DMSO in PBS, $n=5), 1$ and $5 \mu \mathrm{g} / \mathrm{kg} \mathrm{SBF}-$ $1(n=5)$ were intraperitoneally injected to the tumorbearing mice for 14 days. Vehicle and control IgG were administered in 5 days on and 2 days off ( 5 on/ 2 off, QD $\times$ 5). Treatment of nude mice carrying subcutaneous tumors was started when the tumors became palpable and had a mean volume of $50-100 \mathrm{~mm}^{3}$.

\section{Histological assessment}

The samples were isolated and part of the fresh tissues were fixed in $4 \%$ paraformaldehyde and sent for hematoxylin and eosin (H\&E) staining. Sections were photographed by Mantra 1.01 (Perkin Elmer) under 100-fold magnification.

\section{Statistical analysis}

Analysis of experiments with more than two groups was performed using one-way ANOVA with Tukey's correction for multiple comparisons, unless indicated otherwise. For experiments with two groups, statistical analysis was performed using Student's $t$ test with 95\% confidence interval. Statistical analyses in the survival experiments were performed by log-rank (MantelCox) test. Statistical significance is indicated in the figures as follows: ${ }^{*} 0.01 \leq P$ $<0.05$; ${ }^{* * 0} 0.001 \leq P<0.01 ;{ }^{* * *} P<0.001, P$ values $>0.05$ are not indicated.

Other methods: Please see Supplementary Materials and Methods.

\footnotetext{
Acknowledgements

This study was supported by National Natural Science Foundation of China [Nos. 81773743, 81730100]; National Key R\&D Program of China

[2017YFA0506000]; National Science \& Technology Major Project "Key New Drug Creation and Manufacturing Program" [2018ZX09201002]; Natural Science Foundation of Jiangsu Province [BK20150590]; and Nanjing University Innovation and Creative Program for PhD candidate [CXCY17-25].

Author details

${ }^{1}$ State Key Laboratory of Pharmaceutical Biotechnology, Nanjing Drum Tower Hospital and School of Life Sciences, Nanjing University, 210023 Nanjing, China. ${ }^{2}$ Department of Oncology, The First Affiliated Hospital with Nanjing Medical University, 210029 Nanjing, China. ${ }^{3}$ School of Pharmacy, Nanjing
} 
University of Chinese Medicine, 210023 Nanjing, China. ${ }^{4}$ State Key Laboratory of Bioorganic and Natural Products Chemistry, Shanghai Institute of Organic Academy, 200032 Shanghai, China

\section{Authors' contributions}

Y.L.S., Q.L., and Q.X. designed the project. Y.L.S. conducted most experiments. Y. L.S., Y.G., and W.J. conducted some experiments related to mice and cell culture. M.M.F. performed IF and flow cytometer. Y.W. planned and conducted clinical study and analyzed the data. CXS performed UFLC-MS analysis. X.L. conducted OCR monitor. L.J.S. and B.Y. provided SBF-1. Y.L.S. wrote the preliminary paper. Q.X. and Q.L. revised and rewrote the paper.

\section{Conflict of interest}

The authors declare that they have no conflict of interest.

\section{Publisher's note}

Springer Nature remains neutral with regard to jurisdictional claims in published maps and institutional affiliations.

Supplementary Information accompanies this paper at (https://doi.org/ 10.1038/s41419-019-1832-6).

Received: 4 April 2019 Revised: 29 June 2019 Accepted: 17 July 2019 Published online: 13 August 2019

\section{References}

1. Ferlay, J. et al. Cancer incidence and mortality patterns in Europe: estimates for 40 countries and 25 major cancers in 2018. Eur. J. Cancer 103, 356-387 (2018)

2. Siegel, R. L. et al. Colorectal cancer statistics, 2017. Cancer J. Clin. 67, 177-193 (2017).

3. Torre, L. A. et al. Global cancer statistics, 2012. Cancer J. Clin. 65, 87-108 (2015)

4. Cidón, E. U. The challenge of metastatic colorectal cancer. Clin. Med. Insights Oncol. 4, 55-60 (2010).

5. Alberts, S. R. et al. Effect of oxaliplatin, fluorouracil, and leucovorin with or without cetuximab on survival among patients with resected stage III colon cancer: a randomized trial. JAMA 307, 1383-1393 (2012).

6. Taieb, J. et al. Oxaliplatin, fluorouracil, and leucovorin with or without cetuximab in patients with resected stage III colon cancer (PETACC-8): an openlabel, randomised phase 3 trial. Lancet Oncol. 15, 862-873 (2014).

7. Allegra, C. J. et al. Bevacizumab in stage II-III colon cancer: 5-year update of the National Surgical Adjuvant Breast and Bowel Project C-08 trial. J. Clin. Oncol. 31, 359-364 (2013).

8. de Gramont, A. et al. Bevacizumab plus oxaliplatin-based chemotherapy as adjuvant treatment for colon cancer (AVANT): a phase 3 randomised controlled trial. Lancet Oncol. 13, 1225-1233 (2012).

9. Kroemer, G. \& Pouyssegur, J. Tumor cell metabolism: cancer's Achilles' heel. Cancer Cell 13, 472-482 (2008).

10. Hanahan, D. \& Weinberg, R. A. Hallmarks of cancer: the next generation. Cell 144, 646-674 (2011).

11. Vander, H. M. G. Targeting cancer metabolism: a therapeutic window opens. Nat. Rev. Drug Discov. 10, 671-684 (2011).

12. DeBerardinis, R. J. \& Thompson, C. B. Cellular metabolism and disease: what do metabolic outliers teach us. Cell 148, 1132-1144 (2012).

13. Hattori, A. et al. Cancer progression by reprogrammed BCAA metabolism in myeloid leukaemia. Nature 545, 500-504 (2017)

14. Wang, T. J. et al. Metabolite profiles and the risk of developing diabetes. Nat. Med. 17, 448-453 (2011)

15. Mayers, J. R. et al. Tissue of origin dictates branched-chain amino acid metabolism in mutant Kras-driven cancers. Science 353, 1161-1165 (2016).

16. Taniguchi, $K$. et al. The valine catabolic pathway in human liver: effect of cirrhosis on enzyme activities. Hepatology 24, 1395-1398 (1996).

17. Rendina, G. \& Coon, M. J. Enzymatic hydrolysis of the coenzyme a thiol esters of beta-hydroxypropionic and beta-hydroxyisobutyric acids. J. Biol. Chem. 225, 523-534 (1957)

18. Shimomura, Y. et al. Purification and partial characterization of 3hydroxyisobutyryl-coenzyme A hydrolase of rat liver. J. Biol. Chem. 269, 14248-14253 (1994).
19. Graff, J. N., Puri, S., Bifulco, C. B., Fox, B. A. \& Beer, T. M. Sustained complete response to CTLA-4 blockade in a patient with metastatic, castration-resistant prostate cancer. Cancer Immunol. Res. 2, 399-403 (2014).

20. Hardie, D. G. AMP-activated/SNF1 protein kinases: conserved guardians of cellular energy. Nat. Rev. Mol. Cell Biol. 8, 774-785 (2007).

21. Egan, D. F. et al. Phosphorylation of ULK1 (hATG1) by AMP-activated protein kinase connects energy sensing to mitophagy. Science 331, 456-461 (2011).

22. Chouchani, E. T. et al. Ischaemic accumulation of succinate controls reperfusion injury through mitochondrial ROS. Nature 515, 431-435 (2014).

23. Stiles, A. R. et al. Successful diagnosis of $\mathrm{HIBCH}$ deficiency from exome sequencing and positive retrospective analysis of newborn screening cards in two siblings presenting with Leigh's disease. Mol. Genet. Metab. 115, 161-167 (2015).

24. Reuter, M. S. et al. HIBCH deficiency in a patient with phenotypic characteristics of mitochondrial disorders. Am. J. Med. Genet. A 164A 3162-3169 (2014).

25. Kimura, S., Noda, T. \& Yoshimori, T. Dissection of the autophagosome maturation process by a novel reporter protein, tandem fluorescent-tagged LC3. Autophagy 3, 452-460 (2007).

26. Bergers, G. \& Hanahan, D. Modes of resistance to anti-angiogenic therapy. Nat. Rev. Cancer 8, 592-603 (2008).

27. Jain, R. K. et al. Biomarkers of response and resistance to antiangiogenic therapy. Nat. Rev. Clin. Oncol. 6, 327-338 (2009).

28. Escudier, B et al. Phase III trial of bevacizumab plus interferon alfa-2a in patients with metastatic renal cell carcinoma (AVOREN): final analysis of overall survival. J. Clin. Oncol. 28, 2144-2150 (2010).

29. Allegra, C. J. et al. Phase III trial assessing bevacizumab in stages II and II carcinoma of the colon: results of NSABP protocol C-08. J. Clin. Oncol. 29, 11-16 (2011)

30. Greening, D. W. et al. Molecular profiling of cetuximab and bevacizumab treatment of colorectal tumours reveals perturbations in metabolic and hypoxic response pathways. Oncotarget 6, 38166-38180 (2015).

31. Schulte, M. L. et al. Pharmacological blockade of ASCT2-dependent glutamine transport leads to antitumor efficacy in preclinical models. Nat. Med. 24 194-202 (2018).

32. Nicklin, P. et al. Bidirectional transport of amino acids regulates mTOR and autophagy. Cell 136, 521-534 (2009).

33. Schottmann, G. et al. A movement disorder with dystonia and ataxia caused by a mutation in the HIBCH gene. Mov. Disord. 31, 1733-1739 (2016).

34. Ferdinandusse, S. et al. HIBCH mutations can cause Leigh-like disease with combined deficiency of multiple mitochondrial respiratory chain enzymes and pyruvate dehydrogenase. Orphanet J. Rare Dis. 8, 188 (2013).

35. Selvakumaran, M., Amaravadi, R. K., Vasilevskaya, I. A. \& O'Dwyer, P. J. Autophagy inhibition sensitizes colon cancer cells to antiangiogenic and cytotoxic therapy. Clin. Cancer Res 19, 2995-3007 (2013).

36. Curtin, J. C. Novel drug discovery opportunities for colorectal cancer. Expert Opin. Drug Discov. 8, 1153-1164 (2013).

37. Choudry, H. A., Pan, M., Karinch, A. M. \& Souba, W. W. Branched-chain amino acid-enriched nutritional support in surgical and cancer patients. J. Nutr. 136, 314S-318SS (2006).

38. Lukey, M. J., Katt, W. P. \& Cerione, R. A. Targeting amino acid metabolism for cancer therapy. Drug Discov. Today 22, 796-804 (2017)

39. Mayers, J. R. \& Vander, H. M. G. Nature and nurture: what determines tumor metabolic phenotypes. Cancer Res. 77, 3131-3134 (2017).

40. DeBerardinis, R. J. \& Chandel, N. S. Fundamentals of cancer metabolism. Sci Adv. 2, e1600200 (2016).

41. Mayers, J. R. et al. Elevation of circulating branched-chain amino acids is an early event in human pancreatic adenocarcinoma development. Nat. Med. 20, 1193-1198 (2014)

42. Ananieva, E. A. \& Wilkinson, A. C. Branched-chain amino acid metabolism in cancer. Curr. Opin. Clin. Nutr. Metab. Care 21, 64-70 (2018).

43. Ananieva, E. A., Powell, J. D. \& Hutson, S. M. Leucine Metabolism in T Cell Activation: mTOR Signaling and Beyond. Adv. Nutr. 7, 798S-805S (2016).

44. Yoshikawa, R. et al. ECA39 is a novel distant metastasis-related biomarker in colorectal cancer. World J. Gastroenterol. 12, 5884-5889 (2006).

45. Tönjes, M. et al. BCAT1 promotes cell proliferation through amino acid catabolism in gliomas carrying wild-type IDH1. Nat. Med. 19, 901-908 (2013).

46. Panosyan, E. H., Lin, H. J., Koster, J. \& Lasky, J. L. In search of druggable targets for GBM amino acid metabolism. BMC Cancer 17, 162 (2017).

47. Zhang, L. \& Han, J. Branched-chain amino acid transaminase 1 (BCAT1) promotes the growth of breast cancer cells through improving mTOR-mediated 
mitochondrial biogenesis and function. Biochem. Biophys. Res. Commun. 486, 224-231 (2017)

48. Zheng, Y. H. et al. BCAT1, a key prognostic predictor of hepatocellular carcinoma, promotes cell proliferation and induces chemoresistance to cisplatin Liver Int. 36, 1836-1847 (2016).

49. Shi, B., Wu, H., Yu, B. \& Wu, J. 23-oxa-analogues of OSW-1: efficient synthesis and extremely potent antitumor activity. Angew. Chem. Int Ed. Engl. 43, 4324-4327 (2004).

50. Kimberly, W. T., Wang, Y., Pham, L., Furie, K. L. \& Gerszten, R. E. Metabolite profiling identifies a branched chain amino acid signature in acute cardioembolic stroke. Stroke 44, 1389-1395 (2013).
51. Rhee, E. P. et al. A genome-wide association study of the human metabolome in a community-based cohort. Cell Metab. 18, 130-143 (2013).

52. Wang, Y. H. et al. Cell-state-specific metabolic dependency in hematopoiesis and leukemogenesis. Cell 158, 1309-1323 (2014).

53. Lissanu, D. Y. et al. Mutations in the SWI/SNF complex induce a targetable dependence on oxidative phosphorylation in lung cancer. Nat. Med. 24 1047-1057 (2018).

54. Wang, W. et al. The inhibition of TDP-43 mitochondrial localization blocks its neuronal toxicity. Nat. Med. 22, 869-878 (2016). 